\title{
LA MITIGAZIONE: TAPPE DI UN ITINERARIO DI RICERCA
}

\author{
MITIGATION: STEPS OF A RESEARCH ITINERARY \\ Claudia Caffi \\ Università di Genova
}

\section{Riassunto}

In questo articolo richiamerò i punti salienti del modello linguistico pragmatico integrato che ruota intorno al concetto multilivellare e multidimensionale di «mitigazione» (Caffi 2001, 2007) focalizzando l'attenzione su alcuni aspetti che sono stati lasciati in ombra o che, pur ribaditi in diverse sedi, non sono stati sufficientemente intesi, e aggiornandoli alla luce delle mie ricerche più recenti. Di un lungo percorso, con diverse digressioni e ritorni, riassumerò in forma distillata alcune tappe fondamentali per accennare infine ad alcune prossime tappe.

Più specificatamente, il presente contributo si articola in tre parti che hanno i seguenti scopi:

1. richiamare alcune definizioni, punti chiave e sfondi teorici alla base del mio modello. Con un'operazione selettiva molto drastica, metterò in risalto i concetti che ritengo fondamentali;

2. richiamare il criterio euristico alla base delle tipologie formali e funzionali proposte in Caffi (2001, 2007);

3. accennare all'estensione delle applicazioni del modello, in diamesia e diafasia, a un genere particolare di testo scritto, il testo scientifico di ricerca biomedica.

Parole chiave: mitigazione, consapevolezza metapragmatica, sfondi teorici, «scopes» astratti, mitigazioni e tipi di testo, mitigazione nel British Medical Journal. 


\section{DEFINIZIONI E PUNTI CHIAVE}

\subsection{La mitigazione nella cornice della competenza metapragmatica}

L'idea generale di mitigazione è strettamente connessa a quella di una consapevolezza metapragmatica, una "metapragmatic awareness» (Caffi, 1994a; Caffi, 2017) che ci consente di adeguare le parole al mondo, con scelte che rendano il nostro dire appropriato alla situazione comunicativa.

Il concetto di appropriatezza va negoziato tra gli interlocutori. L'appropriatezza di una scelta è giudicata in base a criteri linguistici (è il nostro sapere sulla lingua), enciclopedici (è il nostro sapere sul mondo, che comprende l'esperienza di quella data situazione, o frame) e psicologici (è il nostro sapere su noi stessi, e sugli altri). II sapere multiplo che si manifesta nella mitigazione investe zone procedurali della consapevolezza metapragmatica.

Le scelte linguistiche dei parlanti, per essere appropriate, sono modulate. Sono cioè percorse da una corrente retorica, più o meno lieve, e sono valutabili in base a diversi gradi di intensità. La modulazione copre le due direzioni delle variazioni stilistiche, la mitigazione e il rafforzamento. Una prima definizione di mitigazione è quella di un vasto insieme di mezzi e strategie attraverso le quale i parlanti attenuano uno o più aspetti dei loro discorsi. II concetto di mitigazione può essere visto come una specie di ottimizzazione del sistema: i parlanti modulano in direzione attenuativa i loro atti linguistici per rendere più probabile il raggiungimento dei loro scopi e obiettivi perlocutori evitando rischi non necessari. In particolare, la mitigazione riduce le responsabilità enunciative, i conflitti, e i rischi più o meno direttamente legati alla perdita di faccia.

La mitigazione, che copre la direzione attenuativa della modulazione, è una categoria multilivellare e multidimensionale: investe infatti vari aspetti dell'atto linguistico e vari strati della co-costruzione del senso, inclusi quelli emotivi, e varie dimensioni interazionali.

II modello di mitigazione che nei miei lavori ho proposto può definirsi integrato perché connette idee e categorie attinte da àmbiti disciplinari anche lontani fra loro (cf. par. 2). II nucleo teorico fondamentale entro il quale tale modello si situa è la teoria degli atti linguistici (Austin, 1962). Tale teoria è comunque insufficiente a rendere conto delle dinamiche interazionali, per le quali utili spunti mi sono venuti sia dalla ricerca di linguistica testuale e pragmatica (ad. es., Wunderlich, 1976; Meyer-Hermann e Weingarten, 1982; Hübler, 1983), sia dalla psicologia della comunicazione, come mostrerò nel prossimo paragrafo.

\subsection{Esigenze strumentali ed esigenze di identità}

Un'idea di partenza tratta dalla ricerca psico-sociologica alla base del mio modello di mitigazione è che, nell'interazione, i parlanti, con le loro scelte linguistiche radicate nella loro competenza metapragmatica, devono tener conto sia di esigenze pratiche, strumentali, sia di esigenze relazionali, legate alla costruzione di identità. La mitigazione risponde a entrambi i tipi di bisogni interazionali. 
Riprendendo una distinzione di Giles, Scherer, Taylor (1979), ho proposto che le diverse funzioni della mitigazione ruotino intorno a due dimensioni principali, che differiscono per convenzionalità e validità, e precisamente:

(1) la dimensione dell'efficienza interazionale; entro questa dimensione la mitigazione risponde essenzialmente a bisogni strumentali e pratici, agevolando il raggiungimento degli scopi interazionali;

(2) la dimensione della costruzione di identità; entro questa dimensione la mitigazione risponde essenzialmente a bisogni relazionali, essendo funzionale al monitoraggio delle distanze emotive fra gli interlocutori.

Le due dimensioni sono spesso interrelate. Un'unica scelta linguistica mitigata, ad esempio un diminutivo, risponde contemporaneamente a entrambi i tipi di esigenze. Ho spesso proposto, perché chiarisce ottimamente l'idea dei diversi livelli e delle diverse dimensioni coinvolte dalla mitigazione, l'esempio reale di un medico di base che, al momento della prescrizione, dice al suo paziente: «Le do uno sciroppino». Così dicendo/facendo il medico, sul piano pratico, strumentale, con il mezzo morfologico rappresentato dal suffisso diminutivo all'interno dell'indicazione terapeutica, da un lato rende più probabile il raggiungimento del suo scopo, dell'obiettivo perlocutorio. II tratto semantico [-serio] che Dressler e Merlini Barbaresi (1994) considerano inerente ai diminutivi innesca una serie di implicature: attenua la prescrizione terapeutica, quindi implicitamente la gravità della patologia, e quindi la possibile preoccupazione per essa, rendendo più gradevole il compito. Sul piano interpersonale, il medico con questa scelta accentua la famigliarità, l'intimità, sia pur paternalistica, diminuendo una distanza relazionale con il paziente.

\section{SFONDI TEORICI}

II modello pragmatico integrato che è si avvale del concetto ombrello «mitigazione» (Caffi, 2001; Caffi, 2007) ha alcune fonti di ispirazione che menzionerò in rapido elenco nei prossimi paragrafi. Sono: la retorica, la stilistica della langue e del discours, le teorie sistemiche della comunicazione.

\subsection{La retorica}

Le scelte del parlante, si diceva, sono mirate all'appropriatezza. È qui che entra in gioco la retorica classica, lo sfondo teorico più importante del mio modello. II legame tra retorica e pragmatica è stato oggetto di considerazioni in diverse sedi (ad es. in Caffi 2001) e applicato a diversi oggetti di studio'. È la retorica classica a porre il concetto di APTUM, $\pi \rho \varepsilon \pi \mathrm{ov}$, DECORUM, cioè della scelta adeguata in un contesto. Pragmatica e retorica presuppongono

\footnotetext{
${ }^{1}$ La convinzione dell'importanza della connessione fra retorica e pragmatica è stata alla base della ricerca condotta con Annick Paternoster sul Libro del Cortegiano di Baldassar Castiglione (1528), il primo manuale di comportamento italiano (Paternoster e Caffi 2011). Scritto sotto forma di dialogo, // Cortegiano mette in scena dei comportamenti dialogici che fungono da modelli della conversazione civile. La norma fondamentale, etica ed estetica, attinta alla classicità, posta dal Castiglione: è la norma di equilibrio, misura, giusto mezzo, la cui ottemperanza produce la grazia disinvolta e discreta dell'uomo di Corte. Entro questi comportamenti anche conversazionali, alcune strategie stilistico-pragmatiche di mitigazione, che smorzano diversi aspetti del dire, rivestono un ruolo cruciale.

Normas (ISSN: 2174-7245) 
entrambe un soggetto in movimento, un'attività comunicativa finalizzata a scopi e percorsa da emotività.

Alla base dell'idea di mitigazione vi è l'idea di adattamento: la prima è una forma del secondo. II parlante continuamente adatta il suo dire e il suo agire dicendo alla situazione comunicativa e al destinatario. Che comunicando gli interlocutori si adattino continuamente l'uno all'altro, costruendo strada facendo il senso delle loro azioni linguistiche e il consenso rispetto ad esse, è del resto fra i pochi assunti condivisi dalle teorie del linguaggio, assunto che ha radici lontane e ramificate, sia in linguistica sia in sociologia, ma in primo luogo nella retorica classica. È il concetto, omerico e pitagorico, di politropia, del saper parlare in molti modi a seconda dell'interlocutore (sapienza che Omero attribuiva a Ulisse), che ha un analogo conversazionale nel concetto di «recipient design».

Come termine tecnico, il praxeonimo MITIGATIO, analogo a quello di DEMINUTIO nel senso di mitigazione, azione di calmare le passioni, è introdotto nella Rhetorica ad Herennium (4, 38, 50), tra le figure di pensiero. L'autore, di solito identificato con Cornificio (86-82 a.C.), descrive con precisione gli effetti mitiganti della NEGATIO CONTRARII (con un termine superlativo) e si occupa delle ragioni per le quali è opportuno usarla: fuggire l'invidia ed evitare l'odio. Questa la sua definizione: «Deminutio est... quod deminuitur et adtenuatur oratione. Quare quemadmodum ratione in vivendo fugitur invidia, sic in dicendo consilio vitatur odium». (Rhet. Her., 4, 38, 50).

Cicerone, nel De Oratore, oltre a MITIGATIO (3, 30, 118), usa INMINUTIO ('indebolimento') $(3,54$, 207), termine ripreso da Quintiliano nell'Institutio Oratoria (6, 3, 53).

Rinviando a Caffi (2007) e Caffi (2016) per la ricostruzione della storia del concetto, delle sue diramazioni e applicazioni, qui basterà ricordare che esso approda in pragmatica con l'articolo di Fraser (1980). Tra le proposte contenute in tale lavoro, vi è quella di sganciare la mitigazione dalla cortesia legandola piuttosto all'evitamento di seguiti perlocutori sgradevoli. Nei miei lavori ho mantenuto la separazione fra mitigazione e cortesia: la cortesia è un effetto della mitigazione, e solo uno degli effetti possibili, e non garantiti. Dopo oltre vent'anni di dibattito definitizionale su cosa sia la cortesia, e più di recente a scortesia, ancora in assenza di una definizione ampiamente condivisa, ho sempre ritenuto che, invece di chiedersi "cosa" sia la cortesia, sia più opportuno vedere "come» essa è ottenuta, attraverso quali mezzi, a gradi diversi di convenzionalità. ${ }^{2}$

Vale la pena di notare che tra le varie forme di mitigazione e le figure della DEMINUTIO (ad esempio, litote, eufemismo, perifrasi, reticenza) non v'è salto, ma incremento di implicature: le figure evidenziano con particolare chiarezza meccanismi discorsivi generali, ad esempio, la negabilità o l'offrirsi come strategie «off-record» (Brown e Levinson, 1978, 1987) che le rende idonee al processo di negoziazione del senso. In Caffi (1994b) ho proposto di classificare le figure della DEMINUTIO secondo il criterio della diversa ampiezza del movimento

\footnotetext{
${ }^{2}$ Rispetto ai diversi gradi di convenzionalità dei mezzi di realizzazione di uno stesso atto illocutorio rimane utile il rinvio alla scala di indirettezza proposta in Blum-Kulka et al. (1989: 18). 
cooperativo previsto da parte del destinatario: minimo nell'antifrasi catacresizzata, massimo nella reticenza.

L'afferenza della mitigazione alla comunicazione retorica spiega anche la sua natura paradossale, ${ }^{3}$ il suo essere un'arma a doppio taglio. Se un parlante mitiga, implicitamente segnala che c'è qualcosa da mitigare, una potenziale minaccia o negatività. II vantaggio della mitigazione è la deresponsabilizzazione che consente al parlante. È un vantaggio che si spiega tenendo presente una caratteristica della comunicazione analogica nel senso di Watzlawick et al. (1967), la sua ritrattabilità (cf. 2.3.). Lo svantaggio della mitigazione consiste nel rischio che la funzione strumentale e quella di identità interferiscano in modo conflittuale: nell'interazione, un parlante che mitiga si 'mette in scena' come un partner attento, intento a usare tatto, riguardo, considerazione. Questa «messa in scena» può essere interpretata come calcolo, distanza, non-immediatezza. Se un parlante prende troppe distanze dal suo dire diminuisce la sua responsabilità ma rischia di non essere più credibile o efficace.

\subsection{La stilistica: 'come in musica l'uso del pedale'}

\subsubsection{Variazioni di intensità}

La stilistica della langue di Bally (Bally 1925, 1965; Bally 1909, 1963) e quella del discours (la langue attivata da un parlante) di Spitzer (Spitzer 2007, 1922) ${ }^{4}$ sono state importanti fonti di ispirazione per la mia ricerca sulla mitigazione. Da Bally ho ripreso l'idea di intensità legata ai mezzi espressivi e l'idea di un repertorio di mezzi e di strategie codificate entro il quale il parlante compie una scelta, sia essa neutra (mode pur) sia essa marcata sul piano emotivo (mode vécu). Infatti, ho in più sedi proposto la centralità della componente emotiva della mitigazione che, nell'interazione, funge da calibraggio delle distanze relazionali (Caffi, 2002).

Nelle loro prese di parola i parlanti attuano costantemente delle variazioni stilistiche, delle modulazioni, spesso anche lievi, in direzione sia rafforzante sia attenuativa, usando le risorse adeguate alla situazione che attingono dal repertorio della loro lingua naturale e del loro gruppo sociale "come in musica l'uso del pedale» (Spitzer, 1928, 1954: 69). ${ }^{5}$ E a questa metafora ben si può avvicinare l'idea di Terracini di una tonalità espressiva del discorso (Terracini, 1976: 101).

La mia ricerca sulle modulazioni intese come variazioni di intensità di un dato atto linguistico è partita dall'analogia linguaggio-musica (Caffi, 1990). In tale analogia trovo che il seguente passo di Mann risponda in modo mirabile alla domanda su cosa costituisca il TERTIUM COMPARATIONIS, la

\footnotetext{
${ }^{3}$ Pochi autori riconoscono l'aspetto contraddittorio della mitigazione. Tra questi, Briz (2006: 3).

${ }^{4}$ Per un inquadramento generale sulla stilistica rinvio a Segre (1985). Una raccolta di saggi su fenomeni di intensità nell'italiano parlato è Bazzanella e Gili Fivela (2009).

5 Per tornare alla metafora musicale, si tratta della smorzatura, della Dämpfung, l'esecuzione in sordina: «La parola 'smorzatura' richiama subito alla mente il pedale destinato a questo scopo [ottenere un tono smorzato], non l'altro pedale che prolunga e rafforza la sonorità» (Spitzer, 1931, 2004: 99). Per le metafore musicali in Spitzer (1922, 2007) rinvio a Caffi (2007: 34).

Normas (ISSN: 2174-7245) 
norma rispetto alla quale una determinata scelta è valutabile. Mann ne mette in luce il carattere sfuggente:

«Un accordo così, disse «preso in sé, non ha nessuna tonalità [...] II rapporto è tutto. E se vuoi dargli un nome più preciso, chiamalo ambiguità. [..] «Sai che cosa trovo?» disse «Che la musica è l'ambiguità elevata a sistema. Prendi questo o quel suono. Tu lo puoi intendere così o così, lo puoi considerare aumentato dal basso e diminuito dall'alto e, se sei astuto, puoi sfruttare il doppio senso a tuo piacimento». (Mann, 1947, 1988: 63-64).

Il problema del TERTIUM COMPARATIONIS è sempre stato evocato e mai sciolto fino in fondo né in ambito stilistico né in ambito pragmatico. La mia proposta è stata quella di adottare un concetto del tutto empirico di norma costituita e negoziata all'interno dell'interazione: è lo stile adottato dai parlanti nei turni precedenti (cf. 2.3.).

\subsubsection{Forme della mitigazione in Spitzer}

Citando Spitzer che è ritenuto, insieme a Bally, il fondatore della stilistica novecentesca, non posso non menzionare una direzione di ricerca riassumibile sotto l'etichetta "cantiere spitzeriano" e che è collegata in modo sostanziale al tema della mitigazione. ${ }^{6}$ La Lingua italiana del dialogo rappresenta uno studio pragmatico ante litteram dell'italiano parlato (Caffi, 2007) in cui vengono con grande finezza analizzati meccanismi dialogici che oggi rientrerebbero nelle aree di ricerca dell'analisi della conversazione e del discorso, e degli studi sulla cortesia. In particolare Spitzer osserva varie forme di strategie con le quali parlante e ascoltatore si avvicinano o divergono nel dialogo, minimizzando i rischi, e ricorrendo a diversi dispositivi di mitigazione e di non detti.

Addetto all'ufficio della censura austriaca durante la I guerra mondiale, Spitzer, vagliando la corrispondenza dei prigionieri di guerra italiani aveva rilevato che i prigionieri facevano ricorso a una lingua segreta, intenzionalmente criptica e oscura, che permetteva di comunicare allusivamente informazioni proibite e potenzialmente compromettenti e di eludere la censura. Le conversazioni quotidiane ripropongono secondo Spitzer interdizioni e pericoli avvicinabili, per certi aspetti, a quelli imposti dalla censura. I parlanti devono evitare di offendere gli interlocutori con messaggi che potrebbero urtarne la sensibilità. Nello stesso tempo devono cercare di ingraziarsi l'interlocutore, di rabbonirlo, di coinvolgerlo attraverso varie strategie che a buon diritto possiamo chiamare «di mitigazione».

La diade prigioniero-censore opera dunque sullo sfondo della Lingua italiana del dialogo come metafora-guida, modello interpretativo degli scambi discorsivi quotidiani: tutti noi, in misura ora lieve ora stringente, svolgiamo a un tempo entrambi i ruoli, prigionieri di convenzioni sociali e discorsive, se non di interdizioni, di limitazioni che si riflettono sui

\footnotetext{
${ }^{6}$ Dopo aver curato, con Cesare Segre, l'edizione italiana della Italienische Umgangssprache uscita con il titolo Lingua italiana del dialogo (Spitzer 1922, 2007), mi accingo a curare l'edizione italiana delle Umschreibungen des Begriffes Hunger im Italienischen. La stesura dell'Italienische Umgangssprache si intreccia con quella delle Lettere e con quella delle Perifrasi, la cui edizione italiana, con traduzione di S. Albesano e a cura di chi scrive, è in preparazione per II Saggiatore. Da segnalare la nuova edizione delle Lettere dei prigionieri di Guerra italiani, 1915-1918, con traduzione di R. Solmi, a cura di L. Renzi, Milano, II Saggiatore, 2016, con saggi di A. Gibelli, L. Morlino, E. Benella, S. Albesano e una nuova nota linguistica di L.Vanelli.
} 
discorsi e ci spingono a censurarci, e a interpretare i discorsi altrui proiettandovi le stesse limitazioni e non detti.

\subsection{Le teorie sistemiche della comunicazione}

Le teorie dei sistemi (ad es. Bertalanffy, von, 1968) hanno dato molti spunti alla pragmatica specialmente tramite la mediazione del modello di Watzlawick et al. (1967). In particolare, da Watzlawick et al. ho ripreso l'idea della ritrattabilità della comunicazione analogica. Applicata alla mitigazione, tale idea spiega perché il ricorso alla mitigazione è vantaggioso per i parlanti. Infatti, i parlanti, mitigando, possono non solo aprire uno spazio negoziale di attribuzione di senso, ma anche tornare sui loro passi e negare possibili interpretazioni del loro dire.

Più in generale, il concetto chiave delle teorie dei sistemi è quello di adattamento, che è anche concetto chiave della pragmatica, e alla base del concetto di mitigazione. L'idea generale è quella, olistica, di un sistema complesso all'interno del quale interagiscono diversi parametri eterogenei. Perché questa prospettiva sistemica è idonea a spiegare i meccanismi comunicativi? Qual è il vantaggio rispetto a una prospettiva strutturale che pensa il linguaggio attraverso categorie atomistiche? II vantaggio di una prospettiva olistica è che consente un approccio dinamico all'interazione in cui è focalizzato il funzionamento del sistema come rete di relazioni, e la sua organizzazione contestuale.

II funzionamento della mitigazione in un contesto va spiegata in riferimento a:

(1) l'interconnesione e covarianza di diversi parametri interazionali;

(2) la calibrazione del sistema7, introdotto in Watzlawick et al. (1971, §4.44), che ho proposto di pensare in termini di temperatura dell'interazione (Caffi, 2011; Caffi, 2015).

(3) il concetto di contrasto, di scelta marcata e divergente rispetto a un sistema di attese. La possibile divergenza, almeno in parte sovrapponibile alla categoria linguistica di marcatezza, o a quella conversazionale di preferenza, è sia sintagmatica-sequenziale, all'interno di un co-testo, sia paradigmatica, rispetto a una varietà di lingua e a una situazione comunicativa (Caffi, 1992: 274ss.). Operanti sullo sfondo, sono i diversi tipi di sistemi d'attesa rispetto ai quali una data scelta è valutabile.

Gli studi sull'interazione hanno mostrato che essa funziona in larga misura per confronto e per contrasto e che il comportamento inatteso è particolarmente importante, sia cognitivamente sia emotivamente, in quanto richiede di essere interpretato. In Caffi (2015) ho proposto la categoria di 'inatteso' come iperordinata a quella di (s)cortesia. La domanda che così si pone è: rispetto a che cosa un comportamento comunicativo, ad esempio una scelta mitigata, può essere considerato, a qualche grado di intensità, inatteso, configurando un contrasto di qualche tipo?

\footnotetext{
${ }^{7}$ Quando si cambia la calibrazione di un sistema, o la messa a punto di un termostato, regolandolo ad esempio su una temperatura più alta o più bassa, entrano in gioco funzioni a gradino: il funzionamento del sistema nel suo complesso cambia, ma il meccanismo di retroazione negativa, che scatta ad esempio per correggere eventuali fluttuazioni al di sotto della temperatura stabilita, rimane inalterato. Come è noto, nell'àmbito psichiatrico cui la scuola di Palo Alto riconduce la sua ricerca, la psicosi è interpretabile come brusco cambiamento che ricalibra il sistema famigliare.

Normas (ISSN: 2174-7245)| 
In Caffi (2015) ho ripreso il concetto di Sapir (1927: 893) di «schema nucleare di comportamento" (nuclear pattern of behavior). Tale concetto era stato riformulato in Caffi e Janney (1994: 351) come «schema di anticipazione» (anticipatory schema) e precisato in una serie di distinzioni. Infatti, abbiamo sostenuto in quel lavoro, gli schemi di anticipazione possono essere:

(1) schemi di anticipazione linguistici, che vertono sulle aspettative legate all'uso «non marcato» della lingua in un dato contesto, ai vari livelli, dalla scelta lessicale alla costruzione sintattica, dalla pronuncia all'intonazione;

(2) schemi di anticipazione contestuali, che vertono sulle aspettative a proposito del comportamento comunicativo che tipi diversi di parlanti saranno portati ad adottare in relazione alle diverse situazioni in cui si trovano a interagire. Vi confluiscono sia conoscenze globali sui valori, sentimenti, desideri, motivazioni, atteggiamenti interpersonali, ruoli e convenzioni sociali, sia conoscenze specifiche su come un dato tipo di interlocutore tende a comportarsi in una specifica situazione;

(3) schemi di anticipazione co-testuali, che vertono sulle aspettative riguardo al successivo comportamento comunicativo del partner, elaborate sulla base dei comportamenti adottati e negoziati negli scambi precedenti. Ad esempio, sullo sfondo di una sequenza di turni caratterizzati da uno stile formale, un'improvvisa informalità rappresenterà un contrasto; sullo sfondo di una sequenza di turni caratterizzati da uno stile neutro, non-valutativo, un'improvvisa scelta lessicale fortemente connotata in senso valutativo, ad esempio l'uso di un axionimo, rappresenterà un contrasto.

\section{SCOPES DELL'OPERAZIONE MITIGANTE}

La tipologia funzionale che ho proposto in Caffi (2001) attesta diversi tipi di mitigazione a seconda dei diversi àmbiti o raggi d'azione (scopes), cioè delle componenti astratte all'interno dell'atto linguistico su cui la mitigazione verte. Questi àmbiti convergono verso tre fuochi principali: la proposizione (e, al suo interno, il riferimento e la predicazione), l'indicatore di forza illocutoria, e l'origine deittica dell'enunciazione, l'EGO-HIC-NUNC (Bühler, 1983, 1934). In ciascun caso è possibile che venga impiegato simultaneamente più di un tipo di mezzo mitigante e, inversamente, un solo mezzo mitigante può simultaneamente agire su più di un aspetto dell'atto linguistico.

Chiamo «cespugli» (bushes o hedges proposizionali) i primi mitigatori, centrati sulla proposizione, «siepi» (hedges) i secondi, centrati sull'indicatore di forza, «schermi» (shields) i terzi, centrati sull'istanza d'enunciazione e la sua origine deittica. Come ho già precisato, fra questi casi non è possibile tracciare confini netti, dal momento che le diverse componenti dell'atto linguistico non sono nettamente separabili. Inoltre, i tre tipi di mezzi mitiganti sono eterogenei, in quanto, da un lato, i cespugli e le siepi sono espressioni lessicalizzate, a volte markers, spesso non integrati sintatticamente nell'enunciato. Dall'altro lato, negli schermi, non v'è un esplicito operatore di mitigazione e l'operazione attenuativa ha luogo a un livello più profondo e astratto, riguardando la sintassi, ad esempio nella trasformazione passiva, o la morfologia, ad esempio nel passaggio da una prima persona singolare a una prima persona plurale.

Semplificando, si potrebbe sostenere che i cespugli, tipicamente, diminuiscono i gradi di sottoscrizione al contenuto proposizionale, le siepi diminuiscono il grado di sottoscrizione alla forza illocutoria. Inoltre, la prima operazione sfocia nella seconda, essendo la sottoscrizione alla verità nel caso degli atti assertivi (cf. Venier 1991; Schneider 2007) una delle dimensioni, etichettabile ad esempio con «certezza epistemica», dell'atto linguistico. 
Negli schermi si assiste invece a una dislocazione complessiva della responsabilità enunciativa, ad esempio con la messa in scena di un altro enunciatore, diverso da quello attuale, o con la cancellazione dell'istanza di discorso. Ne deriva una complessiva nonsottoscrizione né cognitiva né emotiva dell'atto linguistico compiuto. Mentre cespugli e siepi sono operatori scalari, in quanto operano lungo una scala di gradi, ad esempio, di impegno epistemico (come molti cespugli) o di gradi di assunzione di una delle dimensioni scalari dell'illocuzione (come è il caso delle siepi), gli schermi sono operatori dicotomici (ad es., EGO vs. NON-EGO, NUNC vs. NON-NUNC) centrati direttamente sul nucleo dell'enunciazione, l'origine deittica, il supporto formale della soggettività. Inoltre, mentre i cespugli e le siepi funzionano IN PRAESENTIA, vale a dire sono espressioni lessicalizzate, gli schermi funzionano IN ABSENTIA, per sostituzione. Negli schermi si ha infatti non l'abbassamento di una qualità di una dimensione scalare interazionale ma piuttosto, a un livello più astratto, una collisione fra attese contestuali e co-testuali e la scelta attualizzata, che fa scattare un contrasto.

\section{MITIGAZIONE E TIPI DI TESTO}

\subsection{II raccordo fra teoria e prassi}

Una prospettiva di ricerca che ho sempre avuto a cuore riguarda il ruolo dei mitigatori nel caratterizzare tipi di testi. Tipi testuali differenti richiedono differenti tipi di mitigatori. Più in generale, da sempre ho avuto presente l'esigenza di confrontarmi con dati reali all'interno di specifici activity types (Levinson, 1992) e di contesti sia istituzionali sia quotidiani e simmetrici. Tale esigenza è dovuta a una ragione interna di ordine generale, ed è la necessità di controllare gli strumenti teorici all'interno di specifici contesti comunicativi reali. Questi contesti specifici pongono delle restrizioni sulle aspettative interazionali e richiedono riaggiustamenti della rilevanza e della reciproca gerarchia di principî, massime e inferenze validi per la comunicazione ordinaria.

Nelle mie ricerche iniziali il tipo discorsivo/testuale indagato utilizzando il modello integrato mitigazione è stato il parlato dialogico in contesti terapeutici, consistenti in incontri tra un paziente e il medico di base, e in sedute psicoterapeutiche e psicoanalitiche. In quei contesti asimmetrici la domanda centrale non veniva soltanto da una scelta teorica e metodologica a priori ma anche dai professionisti che la ponevano in questi termini: quali sono le strategie discorsive più efficaci a costruire l'alleanza terapeutica? Attraverso quali scelte discorsive si costruisce l'empatia? Qual è il contributo della mitigazione a creare un clima empatico? La pertinenza della mitigazione rispetto a tale domanda sta essenzialmente nel fatto che l'empatia non può essere detta, ma deve essere inferibile, ricostruibile sulla base di diverse micro-scelte stilistiche e diversi meccanismi interazionali, a gradi diversi di convenzionalità. Nelle mie ricerche (specialmente in Caffi, 2001 e Caffi, 2007) ho cercato di smontare queste domande e di proporre alcune possibili risposte attraverso la microanalisi dei dati dialogici e la costruzione di diverse tipologie di mitigatori.

I patterns della mitigazione sono risultati utili a catturare la specificità di altri tipi testuali, ad esempio il racconto di sogni. In Caffi (2009) ho sostenuto che questo tipo testuale è caratterizzato dall'occorrenza sistematica di mitigatori di approssimazione, i «cespugli», che introducono vaghezza sul contenuto proposizionale. Tali mitigatori in quel particolare tipo di testo fungono da cerniere tra il mondo del racconto e il mondo onirico rievocato. L'uso 
ricorrente di espressioni quali «una specie di», «qualcosa del genere», «praticamente» non segnala un'incertezza strategica ma la difficoltà reale del parlante a designare oggetti e situazione del mondo costruito dal sogno.

\subsection{Forme della mitigazione nel "British Medical Journal"}

Una delle ricerche che sto svolgendo riguarda la mitigazione nei testi scientifici, in particolare nei testi di ricerca biomedica nel British Medical Journal (da qui in avanti, BMJ). Si tratta di un genere testuale che è già stato indagato sotto l'aspetto dell'attenuazione. II riferimento è in particolare alle ricerche di Hyland (1998) e di Markkanen e Schröder eds. (1997) che hanno investigato il ruolo degli hedges ${ }^{8}$ nei testi scientifici e accademici. Un intero studio, Skelton (1997), è stato dedicato al BMJ.

All'interno di un progetto finanziato dal Ministero della Ricerca ${ }^{9}$ con un gruppo di ricercatori a Genova stiamo investigando il ruolo della mitigazione, specialmente dei markers di certezza e incertezza, nei testi scientifici biomedici, in particolare del BMJ. Qui mi limito ad accennare ad alcuni punti salienti e ad alcune prospettive di ricerca.

Il modello della mitigazione, pensato per lo scambio dialogico all'interno di contesti asimmetrici (terapeutici) è applicabile al testo scientifico scritto di ricerca biomedica? E, se sì, come? In primo luogo, il modello della mitigazione è utile a resettare il problema della certezza/incertezza all'interno di una serie di parametri eterogenei. Altri parametri sono infatti rilevanti: ad esempio precisione/vaghezza, distacco/coinvolgimento emotivo, complessiva sottoscrizione enunciativa/deresponsabilizzazione.

Per me è stata ed è una sfida: cosa c'è di più lontano dal parlato dialogico in contesti terapeutici, carico di emotività e colorato di sottili nuances espressive, di un'algida situazione comunicativa come quella instanziata dal testo scientifico di ricerca? Dove è rintracciabile in questo testo il soggetto e la sua emotività, viste le convenzioni del genere testuale che prevede la cancellazione anche grammaticale dell'ego, con il consueto ricorso alle forme impersonali, al passivo con cancellazione dell'agente etc.? Noterò di passaggio che queste forme di cancellazione dell'EGO sono riconducibili a quelli che ho chiamato «schermi attanziali» (cf. lerardi e Stancampiano, 2016).

Una prima considerazione generale: un'analogia tra tipi discorsivi così distanti è data dall'alta "posta in gioco» (l'enjeu di Ghiglione, 1986) di entrambi. Se nelle interazioni terapeutiche la posta in gioco è la salute di uno dei due interlocutori, nel testo scientifico biomedico quale è il BMJ, la posta in gioco è da un lato, verso il contenuto, la valutazione (assessment) di un oggetto, di un tema di ricerca, ad es. su un farmaco, dall'altro, verso gli scriventi, la loro credibilità e il loro prestigio.

\footnotetext{
${ }^{8}$ Nella vastissima letteratura sugli hedges, mi limito a segnalare Kaltenböck et al., eds. 2010, e Diewald 2006.

${ }^{9}$ Si tratta di un progetto di ricerca di interesse nazionale (PRIN 2012, codice CUP D38C 130006 10001) intitolato Certezza e incertezza nella comunicazione scientifica biomedica. Coordinatore scientifico nazionale: Andrzej Zuckowski, Università di Macerata. Primi risultati di questa ricerca sono stati discussi in un Workshop tenutosi all'Università di Genova nell'aprile 2016 (Caffi et al. 2016; Mey, in press). 
Per tornare a una distinzione proposta all'inizio, la mitigazione, come strategia retorica pragmatica, risponde sia a bisogni strumentali, pratici, sia a bisogni di identità, di costruzione di un self. Tali tipi di esigenze sono interconnessi e possono essere coinvolti simultaneamente da un'unica operazione attenuativa. I bisogni strumentali fondamentali del macro-tipo testuale «testo scientifico di ricerca» (biomedico o no) possono essere identificati con una sorta di regola costitutiva ${ }^{10}$ : è la trasmissione di un sapere nuovo, di un contributo originale, basato su dati ed evidenze, e organizzato secondo una linea argomentativa coerente, da parte di un membro competente (e riconosciuto come tale) di una comunità di scientifica. Si tratta di un macro-tipo testuale prevalentemente espositivo, governato dalla retorica specifica del testo scientifico (cf. Fahnestock, 2009). Rispetto a questa trasmissione è necessaria la cautela. II peso specifico di ogni affermazione di un testo scientifico, di ogni testo scientifico, per le sue conseguenze possibili, e le possibili critiche, impone infatti un ponderato dosaggio del grado di certezza e di sottoscrizione. In particolare la cautela è richiesta per il testo scientifico di ricerca biomedico che ha un enorme impatto potenziale, effetti «perlocutori» multipli, a vari livelli e a varie «fasce» di ricezione e ricaduta. Oltre alla comunità scientifica di esperti in senso stretto, si pensi all'industria farmaceutica, agli enti e alle «agenzie» di gestione della salute, e alle diverse interfacce fra queste fasce di destinatari. Nei testi analizzati si rintracciano forme della mitigazione sia verso il contenuto, sia verso lo scrivente, sia verso il destinatario implicito. Si tratta spesso di formule ritualizzate, con l'uso di markers o costrutti specializzati in un tipo di testo in cui l'adesione al contenuto proposizionale e alla forza illocutoria va attentamente calibrata.

La cautela è dunque d'obbligo, è la caution che è interpretabile come folk-category alla quale corrisponde il concetto tecnico «mitigazione» ${ }^{11}$. La cautela come folk category iperordinata è collegabile anche ai bisogni di identità, è cioè interpretabile, per implicatura, come forma di modestia che si contrappone all'esigenza di auto-promozione.

Nei testi del $B M J$ che abbiamo preso in esame i bisogni di identità sono infatti aggregabili intorno ai due poli principali seguenti, pensabili come due principi regolativi della comunicazione scientifica: da un lato è la modestia, dall'altro è l'auto-promozione. Opposto alla necessità di modestia, rituale o no, è il bisogno di riconoscimento, l'aspirazione all'aumento del prestigio e della «faccia positiva», in un face-work spesso ritualizzato.

La mitigazione si gioca entro queste due esigenze contrapposte e segue patterns distribuzionali nella sequenza testuale codificata prototipica del testo scientifico, per la quale il rinvio è al modello riassunto nell'acronimo IMRD (Swales, 1990). Intorno alla prima esigenza, quella della modestia, è organizzata la prima parte dell'articolo, I'Introduction e quella specificatamente prevista nel BMJ, intitolata Strenghts and limitations of study. Ruota

\footnotetext{
${ }^{10}$ L'uso dell'opposizione «regola costitutiva/regola regolativa» non implica l'adesione al modello di atto linguistico proposto in Searle (1969).

${ }^{11}$ Si ricorderà che la cautela veniva posta da Drew e Heritage (1992) come uno dei due tratti caratterizzanti della comunicazione istituzionale. L'altro tratto è l'evitamento emotivo. Drew-Heritage, tuttavia, non ponevano in relazione i due tratti e non spiegavano da quali indizi testuali fossero inferibili. 
invece intorno alla seconda esigenza, quella di auto-promozione, soprattutto la sezione delle Conclusions.

Ora, la caution viene, con mossa retorica tipica del genere "testo scientifico", non solo ammessa, ma anche tematizzata in modo esplicito dagli autori degli articoli esaminati. Nei due esempi seguenti vi è un richiamo esplicito alla cautela:

(1) «However, caution should be exercised in generalizing the results from our review» (BMJ, October 2013 , p. 3)

(2) «Therefore, the results from this cross-sectional analysis should be interpreted with caution» (BMJ, January 2013, p. 4).

Da notare che in questi casi il richiamo esplicito alla cautela è inserito in un costrutto modale deontico («should») che dà all'atto una forza illocutoria prescrittiva/esercitiva, di raccomandazione e avvertimento. Ora, la fisionomia complessiva degli atti compiuti in questi esempi è anche descrivibile nei termini di «face-work», di costruzione del self autoriale. L'atto illocutorio esercitivo prevede infatti, per la sua «felicità», l'adempimento delle condizioni di potere, di autorità, di competenza da parte del parlante, le condizioni $A$ di Austin (Austin, 1962). Nei due esempi precedenti, con l'atto esercitivo e con l'appello diretto alla comunità scientifica, viene costruito un parlante autorevole e particolarmente attento alla dimensione etica della sua ricerca, anche a scapito delle esigenze autopromozionali.

Altre volte la cautela non è direttamente evocata ma viene comunque attuata una operazione cautelativa/attenuativa che ha da un lato come "scope» astratto il contenuto proposizionale e dunque, nella mia categorizzazione, si manifesta nei cespugli, con tipi diversi di approssimatori (cf. lerardi e Stancampiano, 2016), dall'altro ha come «scope» la forza illocutoria e si manifesta con siepi (avverbi modali, verbi modali, formule di presa di distanza, etc.).

Gli esempi seguenti mostrano le oscillazioni tra modestia e autopromozione e l'intreccio tra cautela verso il contenuto, modestia verso i destinatari e attenta costruzione del self autoriale, nella co-varianza di parametri.

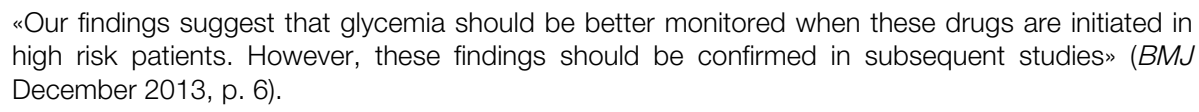

In questo esempio, la scelta lessicale mitigata, «suggest», a un basso grado di evidenzialità, attenua la forza illocutoria della raccomandazione («should be better monitored») a causa della natura non definitiva dei risultati ottenuti, proiettandoli verso uno scenario di più sicura validazione che restringe il campo di indagine a una classe specifica di pazienti. La cautela, verso il contenuto, si accompagna alla modestia verso i risultati raggiunti e verso i lettori. Entrambe le dimensioni traspaiono nell'enunciato finale che, con una mossa rituale della retorica scientifica, rinvia al proseguio della ricerca.

"To our knowledge, this is the first study to evaluate the comparative effectiveness and safety of sitagliptin or any of the DPP-4 inhibitors, at the population level. Given the current controversy about other antidiabetic agents, most notably the thiazolidinediones this is important information for patients and for clinicians managing blood glucose concentrations in patients with diabetes" (BMJ, April 2013, p. 4). 
In quest'ultimo esempio si nota la modulazione operata sul parametro della certezza con l'uso dell'iniziale sintagma limitativo, «to our knowledge», in direzione della cautela. Si tratta di una siepe, di un epistemic disclaimer che da un lato agisce direttamente sul parametro della certezza, dall'altro agisce anche indirettamente, per implicatura, sul parametro della modestia. Questo disclaimer infatti da un lato limita sul piano cognitivo, epistemico, un'asserzione, "this is the first study» troppo categorica; dall'altro, sul piano "emotivo», di costruzione del self, limita la natura auto-promozionale di questa asserzione, di fatto poco modesta. L'operazione "cognitiva» di attenuazione della certezza epistemica sfocia in una operazione «emotiva», di «face-work». L'esempio mostra come le due dimensioni, quella cognitiva epistemica e quella emotiva e di costruzione dell'identità autoriale, siano intrecciate. L'esempio mostra anche l'oscillazione tra le esigenze di modestia e quelle di autopromozione. Dopo avere limitato la portata dell'affermazione sul carattere pionieristico della ricerca proposta, viene ribadita la sua importanza per un insieme specifico di destinatari.

Dalla ricerca, ancora in corso, attraverso lo studio dei patterns della mitigazione, ci aspettiamo di mettere a fuoco dei meccanismi pragmatici multidimensionali che facciano luce sul funzionamento dell'articolo di ricerca biomedica e siano di utilità sia agli autori sia ai fruitori di questo tipo testuale. I risultati ottenuti su forme e funzioni dei markers e delle strategie di mitigazione si presteranno anche a un confronto interlinguistico e interculturale sugli stili espositivi e argomentativi nei testi scientifici.

\section{CONCLUSIONI}

In questo contributo ho ricordato alcuni punti di partenza, alcune tappe e alcune prospettive di un lungo percorso di ricerca sulla mitigazione. Ho richiamato alcuni insieme di rilevanze che hanno consentito l'emergenza dell'oggetto complesso che mi sta a cuore: l'agire linguistico contestualmente modulato ed emotivamente (dis)investito. Per approfondirne ulteriormente lo studio è necessario un raccordo sempre più stretto da un lato, per così dire, in verticale, tra ricerca teorica e base empirica, dall'altro, per così dire, orizzontalmente, tra i diversi àmbiti disciplinari che sono chiamati in causa. Questi due tipi di raccordo non sono affatto scontati. Per quanto riguarda il primo tipo di raccordo: sempre più numerose sono le ricerche "quantitative» ad esempio sui corpora, anche in prospettiva interculturale, che squadernano numeri impressionanti di dati, ma che tuttavia sono prive di un solido nucleo concettuale e di un chiaro orizzonte teorico. E, viceversa, sono numerose le ricerche, ad esempio in ambito di psicologia cognitiva, esclusivamente basate sull'elaborazione concettuale e sull'introspezione. Per quanto riguarda il secondo tipo di raccordo: rinunciare a costruire ponti interdisciplinari, rinunciare cioè alla complessità, significa rinunciare al nostro oggetto, che è complesso, ed è la costruzione discorsiva dell'identità.

\section{RIFERIMENTI BIBLIOGRAFIC}

Austin Langshaw, John (1962): How to Do Things with Words, London, Oxford U.P., London. Trad. in italiano, Come fare cose con le parole, Genova, Marietti, 1987.
Bally, Charles (1965 [1925]): Le langage et la vie, Genève, Librerie Droz.

Bally, Charles (1963 [1909]): Traité de stylistique française. 2 vols., Genève, Librairie de I'Université Georg \& Cie S.A. 
Bazzanella, Carla e Barbara Gili Fivela, a cura di (2009): Fenomeni di intensità nell'italiano parlato, Firenze, Franco Cesati Editore.

Bertalanffy, Ludwig von (1968): General System Theory, New York, Braziller.

Blum-Kulka, Shoshana et alii, eds. (1989): Crosscultural pragmatics: Requests and apologies, Norwood, Ablex.

Briz Gómez, Antonio (2006): «Para una análisi semántico, pragmático y sociopragmático de la cortesía atenuadora en España y América», en LEA: Lingüística española actual, 29, 1, 5-40.

Brown, Penelope e Stephen Levinson (1987 [1978]): "Universals in language usage: politeness phenomena», in Goody, Esther, ed., Questions and politeness: Strategies in social interaction, Cambridge, Cambridge U.P. 56-311.

Bühler, Karl (1983 [1934]): Teoria del linguaggio, Roma, Armando.

Caffi, Claudia (1990): Modulazione, mitigazione, litote, in Conte, Maria-Elisabeth et aliii, a cura di, Dimensioni della linguistica, Milano, Angeli, 169-199.

Caffi, Claudia (1994a): Metapragmatics, in Asher, Ronald and J. Simpson, eds., The Encyclopedia of Language and Linguistics, Oxford, Pergamon,. Repr. in K. Brown, ed., 2006, The Encyclopedia of Language and Linguistics, 2nd Edition, Elsevier, Oxford, 82-88.

Caffi, Claudia (1994b): "Antifrasi, atto illocutorio, atto linguistico, atto locutorio, atto perlocutorio, digressione, enfasi, forza illocutoria, ironia, litote, mitigazione, perifrasi, preterizione, reticenza, riassunto", in Gian Luigi Beccaria, a cura di, Dizionario di linguistica, Torino, Einaudi.

Caffi, Claudia (2001): La mitigazione. Un approccio pragmatico alla comunicazione nei contesti terapeutici, Münster, LIT Verlag.

Caffi, Claudia (2007): Mitigation, Amsterdam / Tokio, Elsevier.

Caffi, Claudia (2009): «Aspetti pragmatici e testuali del racconto di sogni», in Federica Venier, a cura di, Tra pragmatica e linguistica testuale. Ricordando M.-E- Conte, Alessandria, Edizioni dell'Orso, 31-66.

Caffi, Claudia (2015): «(Un)expected behavior: Some general issues and a papal example», Journal of Pragmatics, 86, 19-24.

Caffi, Claudia (2017): "Rethinking metapragmatics: 'What are you talking about?'”, in Allan, Keith et alii, eds., Pragmemes and theories of language use. Cham, Springer, 799-822.

Caffi, Claudia et alii (2016): «Mitigating devices and strategies in the "British Medical Journal"», paper presented in the
Workshop on Certainty and Uncertainty in Biomedical Scientific Communication, University of Genoa, 27 April 2016.

Caffi, Claudia e Richard W. Janney (1994): «Toward a pragmatics of emotive communication", in Caffi, Claudia e Richard W. Janney, eds., Involvement in language, special issue of Journal of Pragmatics, 22, 325-373.

Diewald, Gabriele (2006): "Hecken und Heckenausdrücke. Versuch einer Neudefinition", in Calaresu, Emilia et alii, eds., Italienisch und Deutsch als Wissenschaftssprachen. Bestandsaufnahmen, Analysen, Perspektiven. Italiano e tedesco come lingue della comunicazione scientifica. Ricognizioni, analisi e prospettive, Münster, LIT, 295-315.

Dressler, Wolfgang e Lavinia Merlini Barbaresi (1994): Morphopragmatics, Berlin-New York, de Gruyter.

Drew, Paul, John Heritage (1992): «Analyzing talk at work: an introduction ", in Drew, Paul and John Heritage, eds., Talk at work. Interaction in institutional settings. Cambridge, Cambridge U.P., 3-65.

Fahnestock, Jeanne (2009): «The rhetoric of natural sciences", in The Sage Handbook of rhetoric studies, Los Angeles, Sage, 175-195.

Fraser, Bruce (1980): «Mitigation», Journal of Pragmatics, 4, 341-350.

Ghiglione, Rodolphe (1986): L'homme communiquant, Paris, Colin.

Giles, Howard et alii (1979): "Speech markers in social interaction», in Scherer, Klaus and Howard Giles, eds., Social markers in speech, Cambridge, Cambridge U. P., 343-381.

Hübler, Axel (1983): Understatements and hedges in English, Amsterdam / Philadelphia, Benjamins.

Hyland, Ken (1998), Hedging in scientific discourse, Amsterdam /Philadelphia, Benjamins,

lerardi, Silvia e Daniele Stancampiano (2016), "L'attenuazione nel "British Medical Journal"", paper presented in the International Conference on Linguistic Attenuation: Semantic and Pragmatic Perspectives (CIAL), Valencia, 15-18 giugno 2016,.

Kaltenböck, Gunther et alii, eds. (2010): New approaches to hedging, Emerald, Bingley.

Levinson, Stephen (1992): "Activity types and language", in Drew, Paul and John Heritage, eds., Talk at work. Interaction in institutional settings. Cambridge, Cambridge U.P, 66-100.

Markkanen, Raija e Hartmut Schröder (1997): «Hedging: A challenge for pragmatics and discourse analysis", in Markkanen, Raijna and Hartmut Schröder, eds., Hedging and discourse. Approaches to the analysis of a pragmatic 
phenomenon in academic texts, Berlin / New York, Mouton de Gruyter, 3-18.

Mey, Jacob L., in press, "When hedging fails. Evidentials and (Un)certainty», paper presented at the Workshop on Certainty and Uncertainty in Biomedical Scientific Communication, Genoa University, Italy, 27 April 2016.

Meyer-Hermann, Reinhard e Rüdiger Weingarten (1982): «Zur Interpretation und interaktiven Funktion von Abschwächungen in Therapiegesprächen", in Detering, J. Klaus et alii, eds., Sprache erkennen und verstehen. Akten des 16. Linguistischen Kolloquiums Kiel 1984. Band 2, Tübingen, Niemeyer, 242-252.

Paternoster, Annick e Claudia Caffi (2011): «Una certa sprezzatura". Forme della mitigazione nel Libro del Cortegiano di Baldassar Castiglione (1528)», in Held, Gudrun e Uta Helfrich, eds., Cortesia - Politesse - Cortesía. La cortesia verbale nella prospettiva romanistica. La politesse verbale dans une perspective romaniste. La cortesía verbal desde la perspectiva romanística, Frankfurt am Main / Berlin / Bern / Bruxelles / New York / Oxford / Wien, Narr, 253-274.

Sapir, Edward (1927): "Speech as a personality trait», American Journal of Sociology, 32, 892905.

Schneider, Stefan (2007): Reduced parenthetical clauses as mitigators. A corpus study of spoken French, Italian and Spanish, Amsterdam / Philadelphia, Benjamins.

Searle, John R. (1969): Speech acts. An essay in the philosophy of language, Cambridge, Cambridge U. P. Trad. in italiano Atti linguistici. Saggio di filosofia del linguaggio, Torino, Boringhieri, 1976.

Segre, Cesare (1985): "Stile», in Segre, Cesare, Avviamento all'analisi del testo letterario, Torino, Einaudi, 307-330.

Skelton, John (1997): "How to Tell the Truth in "The British Medical Journal": Patterns of Judgement in the 19 and 20Centuries", in Markkanen, Raijna and Hartmut Schröder, eds., Hedging and discourse. Approaches to the analysis of a pragmatic phenomenon in academic texts, Berlin / New York, Mouton de Gruyter, 42-63.

Spitzer, Leo (1954 [1928]): "Stilistica e linguistica», in Critica stilistica e storia del linguaggio, Bari, Laterza.

Spitzer, Leo (2004 [1931]): "La smorzatura classica nello stile di Racine», in Spitzer, Leo, Saggi di critica stilistica, Firenze, Sansoni, 97227.

Spitzer, Leo (2007 [1922]): Lingua italiana del dialogo, Milano, II Saggiatore.
Spitzer, Leo, (1920): Umschreibungen des Begriffes 'Hunger' im Italienischen, Halle, Niemeyer. Trad. in italiano di Albesano, Silvia, Caffi, Claudia, a cura di, (in preparazione), Le perifrasi del concetto di fame in italiano, II Saggiatore, Milano.

Swales, John (1990): Genre Analysis: English in academic and research settings, Cambridge, Cambridge U.P..

Terracini, Benvenuto (1976): «La speculazione teoretica dei linguisti», in Terracini, Benvenuto, I segni e la storia, Napoli, Guida Editori, 93-104.

Venier, Federica (1991): La modalizzazione assertiva, Milano, Angeli.

Watzlawick, Paul et alii (1967): Pragmatics of human communication, New York, Norton.

Wunderlich, Dieter (1976): Studien zur Sprechakttheorie, Frankfurt a. M., Suhrkamp. 